\title{
Universal level-spacing statistics in quasiperiodic tight-binding models
}

\author{
Uwe Grimm ${ }^{1}$, Rudolf A. Römer ${ }^{1}$, Michael Schreiber ${ }^{1}$, Jian-Xin Zhong ${ }^{2,3}$ \\ ${ }^{1}$ Institut für Physik, Technische Universität, D-09107 Chemnitz, Germany \\ ${ }^{2}$ Department of Physics, University of Tennessee, Knoxville, Tennessee 37996, USA; and \\ Solid State Division, Oak Ridge National Laboratory, Oak Ridge, Tennessee 37831-6032, USA \\ ${ }^{3}$ Department of Physics, Xiangtan University, Xiangtan 411105, P. R. China
}

August 4, 1999

\begin{abstract}
We study statistical properties of the energy spectra of two-dimensional quasiperiodic tight-binding models. The multifractal nature of the eigenstates of these models is corroborated by the scaling of the participation numbers with the systems size. Hence one might have expected "critical" or "intermediate" statistics for the level-spacing distributions as observed at the metal-insulator transition in the three-dimensional Anderson model of disorder. However, our numerical results are in perfect agreement with the universal level-spacing distributions of the Gaussian orthogonal random matrix ensemble, including the distribution of spacings between second, third, and forth neighbour energy levels.
\end{abstract}

Keywords: quasicrystals; electronic properties; tight-binding model; multifractality; energy-level statistics; random matrix theory

\section{Introduction}

The anomalous transport properties of quasicrystals evince the peculiar electronic properties of these intermetallic alloys, which are reflected in the nature and the density of electronic states in quasiperiodic systems. Tight-binding models on quasiperiodic tilings serve as simple toy models of electrons moving in a quasiperiodic environment. While much is known rigorously for one-dimensional aperiodic Schrödinger operators [1], quasiperiodic tightbinding models in the physically relevant dimensions two and three have mainly been investigated numerically, compare [2] and references therein for the few exceptions, which, however, yield only rather limited information about the overall structure of the spectrum or the wave functions.

At least in two dimensions, numerical results provide ample evidence that typical eigenstates of quasiperiodic tight-binding Hamiltonians are, as in the one-dimensional case, neither extended nor exponentially localized; instead, the probability amplitudes show a multifractal distribution [3]. However, the integrated density of states does not show the hierarchical plateau structure observed for onedimensional substitution chains [4]; for pure hopping Hamiltonians it appears to be rather smooth, apart from a few gaps in the spectrum. This makes it possible to employ spectral statistics [6] as further tools to characterize the energy spectrum of quasiperiodic tight-binding models.

\section{Energy-level statistics}

The statistical analysis of the spectra of quantum systems has a rich history, and is at the center stage of current interest [5]. For disordered systems, it is by now well known that in the metallic regime the level-spacing distribution $P_{0}(s)$ of neighbouring eigenenergies is well described by $P_{0}^{\mathrm{GOE}}(s)$ of the Gaussian orthogonal ensemble (GOE) of random matrix theory [6]. Here, $s$ denotes the energy spacing in units of the mean level spacing, and $P_{0}(s)$ gives the distribution of normalized gaps in the spectrum in the limit of infinite system size. In the insulating regime, the level-spacing distribution follows the Poisson law $P_{0}^{\mathrm{P}}(s)=\exp (-s)$. At the metal-insulator transition, the level-spacing distribution has been shown to follow yet another behavior which is attributed to the existence of a "critical ensemble" [7, 8, 9.

Recently, we have shown that the level statistics of tight-binding models defined on planar quasiperiodic tilings, notably the Ammann-Beenker tiling shown 

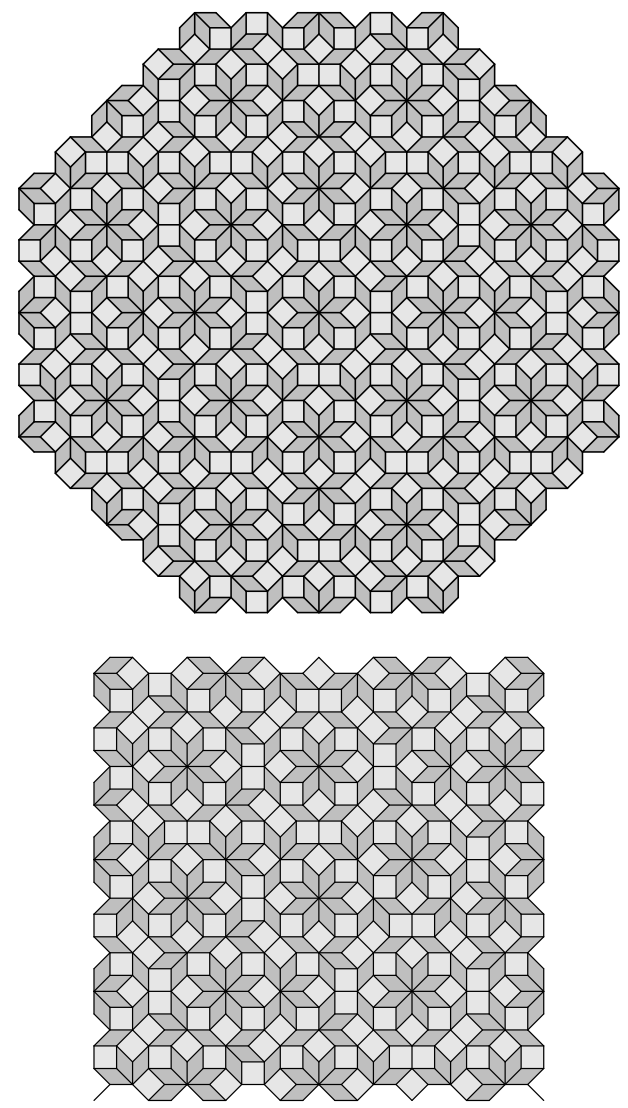

Fig. 1. Top: Octagonal cluster of the Ammann-Beenker tiling with 833 vertices and exact $D_{8}$-symmetry around the central vertex. Bottom: A square-shaped cut with 496 vertices without any exact spatial symmetries.

in Fig. 1, is also given by the GOE predictions 10, contrary to previous statements in the literature 11, 12, 13]. The deviations from GOE behaviour observed in 11, 12 could be understood by noting that the standard periodic approximants of the octagonal tiling are singular patches whose fourfold rotational symmetry is broken only weakly, see Fig. 2. This "almost symmetry" of the patch does not lead to a block structure of the Hamiltonian (which would mean independent spectra for each block), but nevertheless affects the level-spacing distribution. As shown in [10], the level-spacing distribution $P_{0}(s)$ of generic patches of the tiling, or of the irreducible sectors of patches with exact symmetries, follows the GOE distribution when applying a suitable unfolding procedure to correct for the fluctuations in the density of states. This result was further corroborated by higher correlations such as the Mehta-Dyson statistics $\Delta_{3}$ [10] and the number variance $\Sigma_{2}$ [14]. Furthermore, we have shown that the level-spacing distribution remains GOE if one considers small parts of the spectrum without any unfolding, and we demonstrated that the unfolding procedure has to be performed somewhat differently from the procedure in

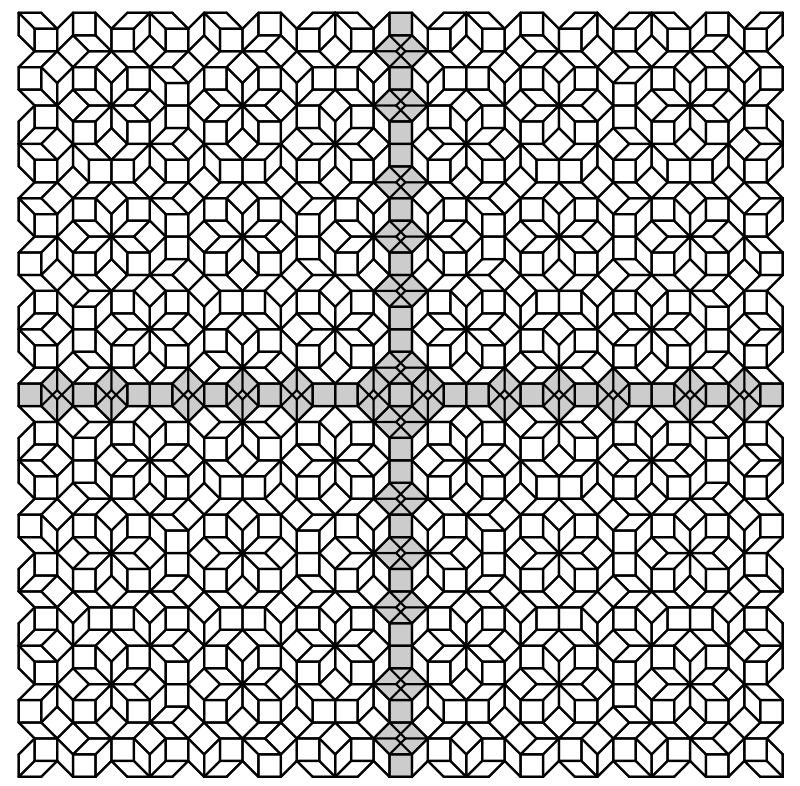

Fig. 2. Periodic approximant of the Ammann-Beenker tiling with 1393 vertices, displayed together with a copy of itself that has been rotated through 90 degrees. The "worms" where mismatches between the two rotated copies occur are shaded.

disordered systems 15 .

In this paper, we numerically calculate not only the first, but also the second, the third, and the fourth neighbour level-spacing distributions $P_{n}(s)$, $n=0, \ldots, 3$, respectively. We show that the higher level-spacing distributions are also well described by the predictions of random matrix theory. The recently proposed "semi-Poisson ensemble" [16], supposed to be valid for the multifractal eigenstates of the metal-insulator transition in the three-dimensional Anderson model [17, does not describe the higher level-spacing distributions of the quasiperiodic models. The same applies to other intermediate statistics obtained recently for an ensemble constructed by an analogy with a one-dimensional gas model of particles with nearest neighbour interactions $V(x)=-\log |x||| 18 \mid$. This is intermediate between the Poisson and the GOE statistics. which can also be interpreted in terms of Boltzmann distributions of one-dimensional interacting gases with pair potentials $V(x)=0$ and $V(x)=-\log |x|$, but not restricted to nearest neighbours, respectively. Finally, we calculate the participation numbers in these tilings and show that their scaling behavior indicates multifractal behavior.

\section{Higher level-spacing distributions}

In addition to the nearest-neighbour level-spacing distribution $P_{0}(s)$ considered previously 10, 14, 15], we have now computed the distributions $P_{1}(s)$, 


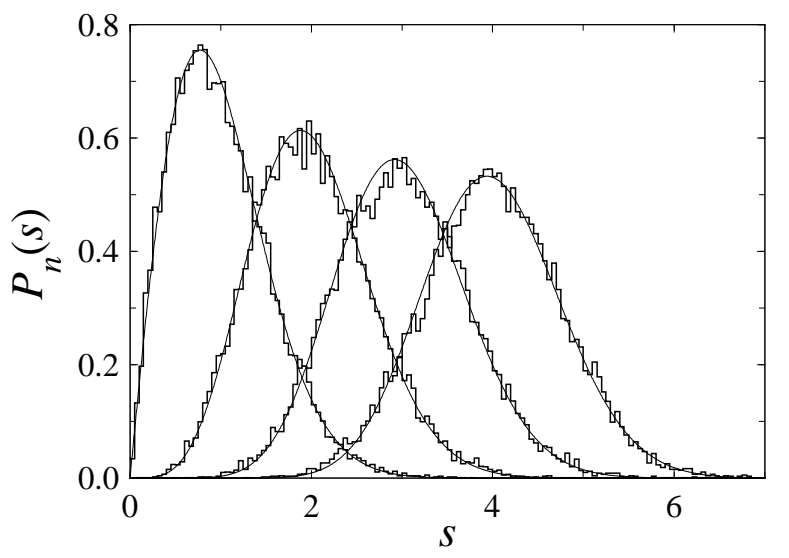

Fig. 3. Level-spacing distribution up to the forth neighbour spacing for the largest sector of the octagonal patch with $N=157369$ vertices. The thin lines represent the GOE predictions [19].

$P_{2}(s)$, and $P_{3}(s)$ for the simple Hamiltonian

$$
H=\sum_{i j}^{N} t_{i j}|i\rangle\langle j|
$$

defined on the Ammann-Beenker or octagonal tiling as shown in Fig. 1. Here, each vertex $i=1,2, \ldots, N$ of the tiling carries a state $|i\rangle$, and the hopping elements $t_{i j}=t_{j i}$ are chosen to be unity between neighbouring vertices, i.e. vertices connected by a bond, and zero otherwise. The spectral statistics are obtained by numerically diagonalizing the Hamiltonian (1) with free boundary conditions for large patches of the tiling, taking into account their symmetries. While the GOE predictions for these distributions are known in principle [6], it is non-trivial to obtain numerical data for these functions. As we could not find sufficiently accurate results in the literature, we calculated $P_{0}^{\mathrm{GOE}}(s), P_{1}^{\mathrm{GOE}}(s), P_{2}^{\mathrm{GOE}}(s)$, and $P_{3}^{\mathrm{GOE}}(s)$ using the approach of 19 by numerically solving the appropriate differential equations. This was done using Mathematica by performing a small-s expansion, followed by a numerical integration to larger values of $s$. The small- $s$ behaviours of the GOE distributions are $P_{0}^{\mathrm{GOE}}(s)=$ $\pi^{2} s / 6+\mathcal{O}\left(s^{3}\right), P_{1}^{\mathrm{GOE}}(s)=\pi^{4} s^{4} / 270+\mathcal{O}\left(s^{6}\right)$, $P_{2}^{\mathrm{GOE}}(s)=\pi^{8} s^{8} / 1764000+\mathcal{O}\left(s^{10}\right)$, and $P_{3}^{\mathrm{GOE}}(s)=$ $\pi^{12} s^{13} / 168781725000+\mathcal{O}\left(s^{15}\right)$. In our calculations we evaluated the expansions beyond the 30th order.

In Fig. 3, we show the results for the data of the largest patch considered in 10], an octagonal patch as in Fig. 1 but with 157369 vertices, employing the unfolding procedure described in 15]. Apparently, our data agree very well with the GOE distributions. We like to emphasize that the higher level-spacing distributions considered here, and in particular their small- $s$ behaviour, are very sensitive to detect devia-

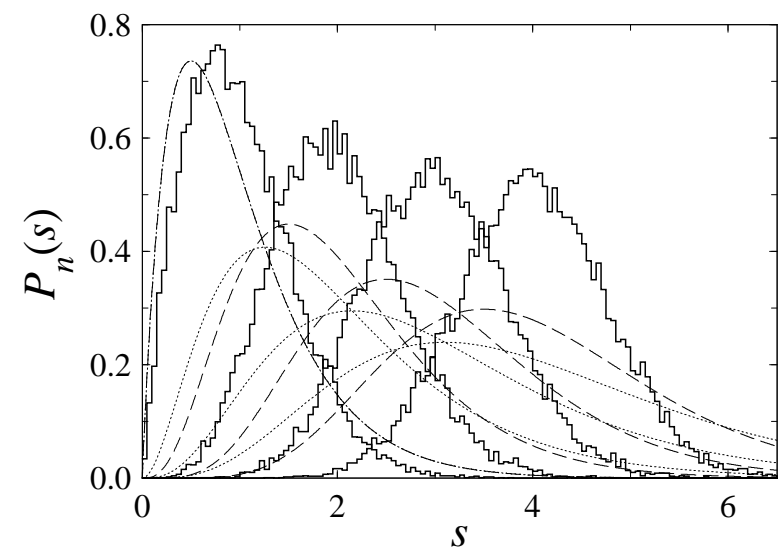

Fig. 4. Comparison of the level-spacing distributions of Fig. 3 with the results for the semi-Poisson (dotted) and the one-dimensional gas model (dashed) ensembles. Note that $P_{0}(s)$ is identical for these two intermediate ensembles.

tions from the GOE behaviour. However, we do not find deviations beyond the fluctuations expected for finite systems. Thus, the level-spacing distribution is the same as that of the Anderson model of localization in the metallic regime [7, 8]. This is surprising, because it is well-known that eigenstates in the metallic regime are extended, whereas there is ample evidence that the eigenstates on planar quasiperiodic tilings have a multifractal character. Thus one might speculate that the level statistics should have been similar to the intermediate statistics found 17 at the metal-insulator transition in three dimensions, where eigenstates are multifractal as well. In Fig. A, we compare our results to the level-spacing distributions of the intermediate semi-Poisson ensemble [16. 18] and the one-dimensional gas ensemble with logarithmic interactions [18]. These latter statistics clearly do not agree with our data.

\section{Scaling of the participation numbers}

We now turn our attention to the calculation of the participation numbers $P(N, E)$ in order to investigate the properties of the states in the tiling directly. For this purpose, we consider square-shaped patches as in Fig. 1 with up to $N=7785$ vertices [10] and compute

$$
P(N, E)^{-1}=\sum_{i=1}^{N}\left|\psi_{i}^{4}(E)\right|
$$

where $\psi(E)=\left(\psi_{1}(E), \ldots, \psi_{N}(E)\right)$ denotes a normalized eigenstate at energy $E$. Thus a state $\psi(E)$ that is completely localized at site $j$, i.e. $\psi_{i}(E)=\delta_{i, j}$, has $P(N, E)=1$, whereas a fully extended state $\psi_{i}(E)=1 / \sqrt{N}$ gives $P(N, E)=N$. Because for 


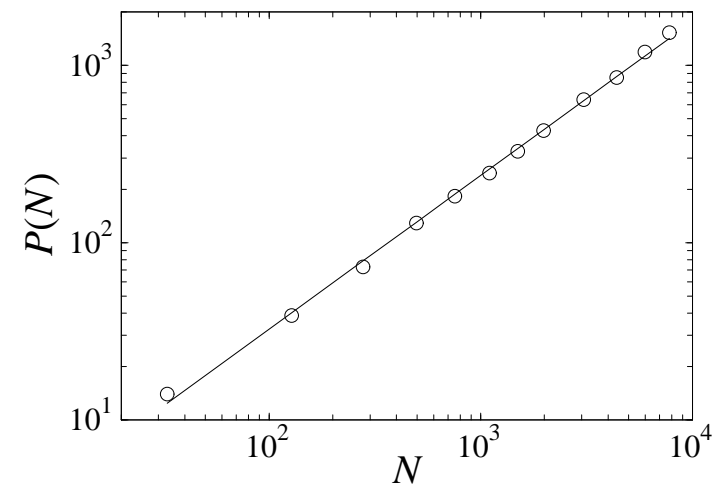

Fig. 5. Energy-averaged participation numbers versus system size for square-shaped patches (cp. Fig. 1). The straight line is a least-squares fit of $\log P(N)=\kappa \log N+c$ to the data.

the models under consideration the level-spacing distribution does not appear to depend on the energy 15], we concentrate on the energy-averaged participation number $P(N)$. For multifractal states, we expect to find a power-law behaviour $P(N) \sim N^{\kappa}$ as a function of systems size [20], between the result for localized $(\kappa=0)$ and extended $(\kappa=1)$ states. The result is shown in Fig. 5, which yields a value of $\kappa \approx 0.87 \pm 0.05$. This value, in agreement with previous results for quasiperiodic tight-binding models [3], is clearly below the values $\kappa=1$ for extended states, albeit rather close, corroborating the multifractal character of the eigenstates in our model, though with rather large characteristic exponents [3].

\section{Conclusions}

We considered the spectral properties of a tightbinding Hamiltonian defined on the AmmannBeenker tiling. Although this system is not energetically disordered, the level-spacing distributions exhibit level repulsion and are in perfect agreement with the GOE predictions. This is similar as for the energetically disordered Anderson model of localization in the metallic regime [8], and also for tightbinding models defined on certain random graphs [21. Nevertheless, the scaling of the energy-averaged participation numbers confirms that the corresponding eigenstates are not extended. Their multifractal character is further corroborated by the system-size dependence of the participation numbers. At first glance, it appears surprising that multifractal states may give rise to GOE level statistics. However, it is known from studies of the metal-insulator transition in more than two dimensions that the critical level statistics becomes similar to the Poissonian behaviour for larger dimensions [22]. Thus, it appears possible that the critical level statistics for the twodimensional case considered here just coincides with the Gaussian orthogonal random matrix ensemble. This scenario is in perfect agreement with the results presented here.

\section{Acknowledgements}

We thank C. A. Tracy for pointing out reference 119 and for suggesting how to compute $P_{n}^{\mathrm{GOE}}(s)$. We also thank U. Gerland and E. B. Bogomolny for stimulating discussions on intermediate statistics.

\section{References}

[1] A. Sütő, Schrödinger difference equation with deterministic ergodic potentials, in: Beyond Quasicrystals, F. Axel and D. Gratias (Eds.), Springer, Berlin and Les Éditions de Physique, Les Ulis, 1995, pp. 481-549.

[2] P. Repetowicz, U. Grimm, M. Schreiber, Exact eigenstates of tight-binding Hamiltonians on the Penrose tiling, Phys. Rev. B 58 (1998) 13482-13490.

[3] T. Rieth, M. Schreiber, Numerical investigation of electronic wave functions in quasiperiodic lattices, J. Phys.: Condens. Matter 10 (1998) 783-800.

[4] J. Bellissard, A. Bovier, J. M. Ghez, Gap labelling theorems for one-dimensional discrete Schrödinger operators, Rev. Math. Phys. 4 (1992) 1-37.

[5] M. Schreiber (Ed.), Proceedings of the 210. WE-HeraeusSeminar on Percolation, Interaction, Localization: Simulations of Transport in Disordered Systems, Berlin, October 6-9, 1998, Ann. Phys. (Leipzig) 7 (1998) 343-584.

[6] M. L. Mehta, Random Matrices, 2nd ed., Academic Press, Boston, 1990.

[7] B. I. Shklovskii, B. Shapiro, B. R. Sears, P. Lambrianides, H. B. Shore, Statistics of spectra of disordered systems near the metal-insulator transition, Phys. Rev. B 47 (1993) 11487-11490.

[8] E. Hofstetter, M. Schreiber, Statistical properties of the eigenvalue spectrum of the three-dimensional Anderson Hamiltonian, Phys. Rev. B 48 (1993) 16979-16985; Relations between energy-level statistics and phase transitions and its applications to the Anderson model, Phys. Rev. B 49 (1994) 14726-14729; Does broken time reversal symmetry modify the critical behavior at the metalinsulator transition in 3-dimensional disordered systems? Phys. Rev. Lett. 73 (1994) 3137-3140.

[9] I. K. Zharekeshev and B. Kramer, Asymptotics of universal probability of neighboring level spacings at the Anderson transition, Phys. Rev. Lett. 79 (1997) 717-720.

[10] J. X. Zhong, U. Grimm, R. A. Römer, M. Schreiber, Level-spacing distributions of planar quasiperiodic tightbinding models, Phys. Rev. Lett. 80 (1998) 3996-3999.

[11] V. G. Benza, C. Sire, Band spectrum of the octagonal quasicrystal: Finite measure, gaps, and chaos, Phys. Rev. B 44 (1991) 10343-10345.

[12] F. Piéchon, A. Jagannathan, Energy-level statistics of electrons in a two-dimensional quasicrystal, Phys. Rev. B 51 (1995) 179-184.

[13] J. X. Zhong, H. Q. Yuan, Statistics of energy levels and quantum dynamics in quasiperiodic tilings, in: Proceedings of the 6th International Conference on Quasicrystals, Tokyo, May 26-30, 1997, S. Takeuchi and T. Fujiwara (Eds.), World Scientific, Singapore, 1998, pp. 184-187

[14] M. Schreiber, U. Grimm, R. A. Römer, J. X. Zhong, Application of random matrix theory to quasiperiodic systems, Physica A 266 (1999) 477-80. 
[15] M. Schreiber, U. Grimm, R. A. Römer, J. X. Zhong, Energy levels of quasiperiodic Hamiltonians, spectral unfolding, and random matrix theorv. Comp. Phys. Commun. (to appear, preprint cond-mat/9811359).

[16] E. B. Bogomolny, U. Gerland, C. Schmit, Models of intermediate spectral statistics, Phys. Rev. E 59 (1999) R1315-R1318.

[17] D. Braun, G. Montambaux, M. Pascaud, Boundary conditions at the mobility edge, Phys. Rev. Lett. 81 (1998) 1062-1065.

[18] U. Gerland, Quantum Chaos and Disordered Systems: How close is the analogy? Doctoral thesis, Heidelberg, 1998.

[19] E. L. Basor, C. A. Tracy, H. Widom, Asymptotics of levelspacing distributions for random matrices, Phys. Rev. Lett. 69 (1992) 5-8.

[20] A. Eilmes, R. A. Römer, M. Schreiber, The twodimensional Anderson model of localization with random hopping, Eur. Phys. J. B 1 (1998) 29-38.

[21] U. Grimm, R. A. Römer, G. Schliecker, Electronic states in topologically disordered systems, Ann. Phys. (Leipzig) 7 (1998) 389-393.

[22] I. Kh. Zharekeshev, B. Kramer, Critical level statistics at the Anderson transition in four-dimensional disordered systems, Ann. Phys. (Leipzig) 7 (1998) 442-451. 